\title{
A Study of an Iterative Channel Estimation Scheme of FS-FBMC System
}

\author{
YongJu Won, JongGyu Oh, JinSeop Lee, and JoonTae Kim \\ Konkuk University, Seoul, Republic of Korea \\ Correspondence should be addressed to JoonTae Kim; jtkim@konkuk.ac.kr
}

Received 31 July 2016; Accepted 5 October 2016; Published 1 January 2017

Academic Editor: Pierre Leone

Copyright (C) 2017 YongJu Won et al. This is an open access article distributed under the Creative Commons Attribution License, which permits unrestricted use, distribution, and reproduction in any medium, provided the original work is properly cited.

\begin{abstract}
A filter bank multicarrier on offset-quadrature amplitude modulation (FBMC/OQAM) system is an alternative multicarrier modulation scheme that does not need cyclic prefix (CP) even in the presence of a multipath fading channel by the properties of prototype filter. The FBMC/OQAM system can be implemented either by using the poly-phase network with fast fourier transform (PPN-FFT) or by using the extended FFT on a frequency-spreading (FS) domain. In this paper, we propose an iterative channel estimation scheme for each sub channel of a FBMC/OQAM system over a frequency-spreading domain. The proposed scheme first estimates the channel using the received pilot signal in the subchannel domain and interpolates the estimated channel to fine frequency-spreading domain. Then the channel compensated FS domain pilot is despread again to modify the channel state information (CSI) estimation. Computer simulation shows that the proposed method outperforms the conventional FBMC/OQAM channel estimator in a frequency selective channel.
\end{abstract}

\section{Introduction}

The filter bank multicarrier on offset QAM (FBMC/OQAM) system is an alternative multicarrier modulation which does not need the cyclic prefix $(\mathrm{CP})$ or guard interval in orthogonal frequency division multiplexing on QAM (OFDM/QAM) system [1] which is widely used in mobile communication [2] and digital broadcasting transmission system [3]. In FBMC/OQAM system, designers employ a real prototype filter with good localization properties in the time and frequency domains to deal with the intersymbol interference (ISI) and intercarrier interference (ICI) without CP [4] where the real or imaginary axis subchannels convey the real or imaginary parts of QAM data constellation, respectively, overlapping with half of the QAM data period staggered time offset. An implementation scheme for a filter bank in FBMC/OQAM system is introduced using an extended fast Fourier transform (FFT) module which is called frequencyspreading FBMC (FS-FBMC) in the frequency domain [5]

The prototype filter of FBMC/OQAM system exploits a longer time impulse response and frequency fragmented response compared to a rectangular prototype filter in
OFDM/QAM system [6]. Thus each subchannel in FBMC/ OQAM system overlaps in the time and frequency domains. In spite of overlapping, the data can be perfectly reconstructed at the receiver side by taking the real value of the complex value after filtering; we call this property "real orthogonality" [7]. According to real orthogonality, the data can be reconstructed perfectly after taking the real value from the complex value, after matched filtering at the analysis filter bank in the receiver side.

If FBMC/OQAM signal is received through a fading channel, it can be equalized in the frequency domain with estimated channel state information (CSI), using scattered pilots similar to OFDM/QAM system [8] after cancelling the intrinsic interference at the pilot subchannel.

In this paper, we introduce an iterative channel estimation scheme for each subchannel of a FBMC/OQAM system over a frequency-spreading domain. The proposed scheme first estimates the channel using the received pilot signal in the subchannel domain and interpolates the estimated channel to fine frequency-spreading domain. Then the channel compensated FS domain pilot is despread again to modify the channel state information (CSI) estimation. Computer 


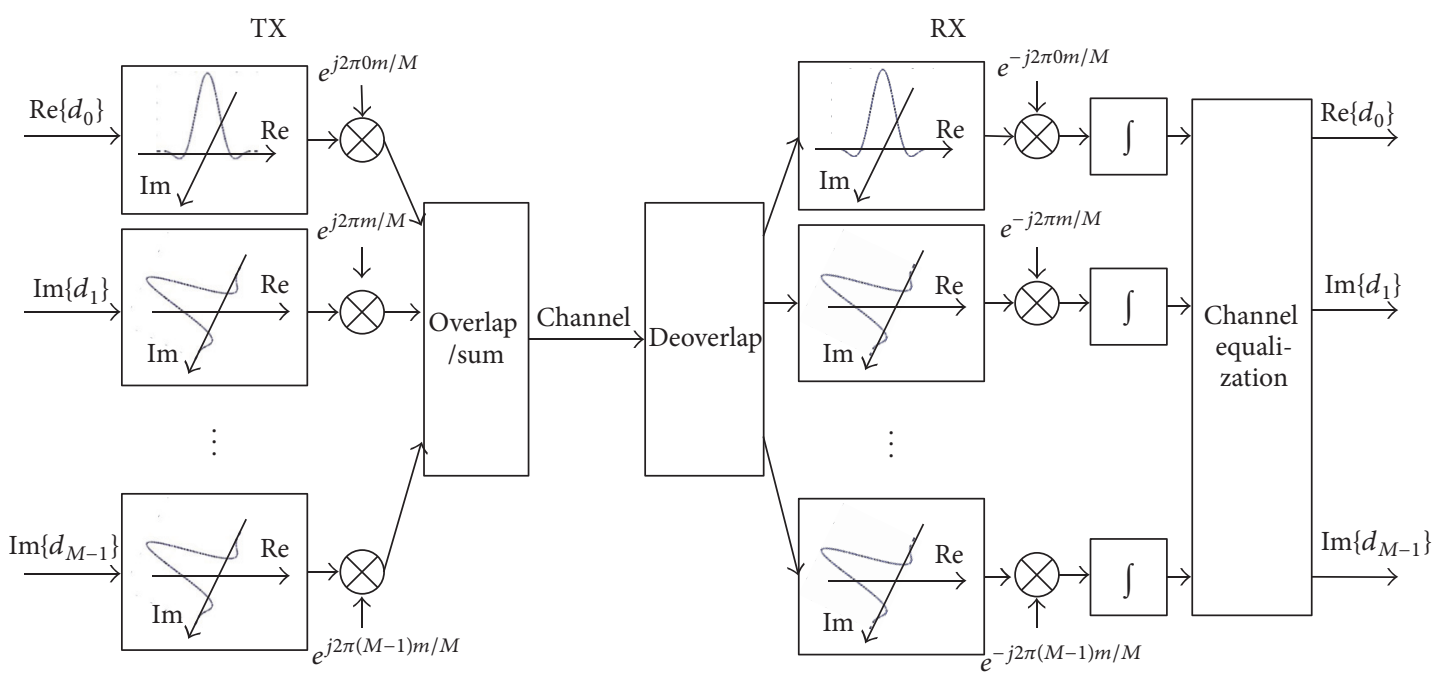

FIgURE 1: The FBMC system.

simulation shows that the proposed method outperforms the conventional FBMC/OQAM channel estimator in a frequency selective channel as the iteration stage increases.

The paper is organized as follows. In Section 2, fundamental FS-FBMC system is explained, and the proposed iterative channel estimation scheme for FS-FBMC/OQAM system is proposed in Section 3. Computer simulation results are included in Section 4.

\section{FS-FBMC System Representation}

Figure 1 illustrates the block diagram of transmitter part and receiver part of FBMC system where real part of complex symbols is conveyed through the even subchannels whereas imaginary part of complex symbols on odd subchannels. Offset QAM is a scheme that the real part and imaginary part separated from a QAM data constellation are transmitted with a half symbol time delay. Thus, the baseband signal of FBMC/OQAM system can be represented as follows [4]:

$$
s(t)=\sum_{m=0}^{M-1} \sum_{n=0}^{N-1} j^{(m+n) \bmod 2} d_{m, n} g\left(t-n \tau_{0}\right) e^{j 2 \pi m f_{0} t},
$$

where $M$ is the number of subchannels; $d_{m, n}$ is the real or imaginary part of the QAM data carried on the $m$ th subchannel in the $n$th FBMC symbol; $f_{0}$ is the spacing between neighboring subchannels in a frequency domain; $\tau_{0}$ is the time offset between FBMC symbols; and $g_{m, n}(t)$ is the filter of the $m$ th subchannel in the $n$th FBMC symbol. $g_{m, n}(t)$ can be obtained from the prototype filter $g(t)$ of the overlapping factor $K=4$, as introduced in [5].

The filter bank of FBMC/OQAM system can be generated by using poly-phase network with inverse FFT process (PPNFFT) [9] in the time domain or frequency-spreading with extended inverse FFT process (FS-FBMC) in the frequency domain. Each approach has different advantage; the PPNFFT approach can reduce the hardware complexity, while FSFBMC approach can achieve high equalization and timing offset compensation performance [10].
In the FS-FBMC approach, an OQAM data constellation is conveyed on several frequency points to the FFT process. Therefore, an OQAM data constellation has to be spread over several frequency points, which correspond to the frequency coefficients of prototype filter $G_{|k|}$ for $(-K+1 \leq k \leq K-1)$, as in Figure 2. In this paper, the frequency coefficients of prototype filter introduced in [5] for $K=4$ are used. This operation is called "frequency-spreading" [5]. The set of frequency points to convey a single OQAM data constellation is called subchannel (i.e., an OQAM data element was carried at each subchannel, the set of frequency points). After frequencyspreading, all frequency points transmitted by using an extended inverse FFT module to generate 1 FBMC symbol.

To reach an equivalent data rate with an OFDM/QAM system without CP, the FBMC symbols are overlapped with half of the QAM data period staggered time offset, which is called as offset QAM scheme. Finally, FBMC/OQAM signal can be generated by these processes in FS-FBMC system.

Assuming a distortion free channel, the data which is transmitted in the $m$ th sub-channel in the $n$th FBMC symbol can be reconstructed after matched filtering with $g_{m, n}^{*}(t)$ in analysis filter bank as follows:

$$
\begin{aligned}
\left\langle s(t) \mid g_{m, n}\right\rangle & =\int_{-\infty}^{\infty} s(t) g_{m, n}^{*}(t) d t \\
& =d_{m, n}+\underbrace{j \sum_{p, q \neq m, n} d_{p, q}\langle g\rangle_{m, n}^{p, q},}_{I_{m, n}}
\end{aligned}
$$

where $\langle g\rangle_{m, n}^{p, q}$ is the interference value from the neighboring subchannel of FBMC/OQAM system, after matched filtering by analysis filter bank at the receiver side. Therefore, the result of (2) comprised the data part $d_{m, n}$ and interference part $I_{m, n}$ from the neighboring subchannels.

The interference part $I_{m, n}$ of (2) remained as imaginary in FBMC/OQAM system. This property is called "real orthogonality" [3]. According to the real orthogonality of the 


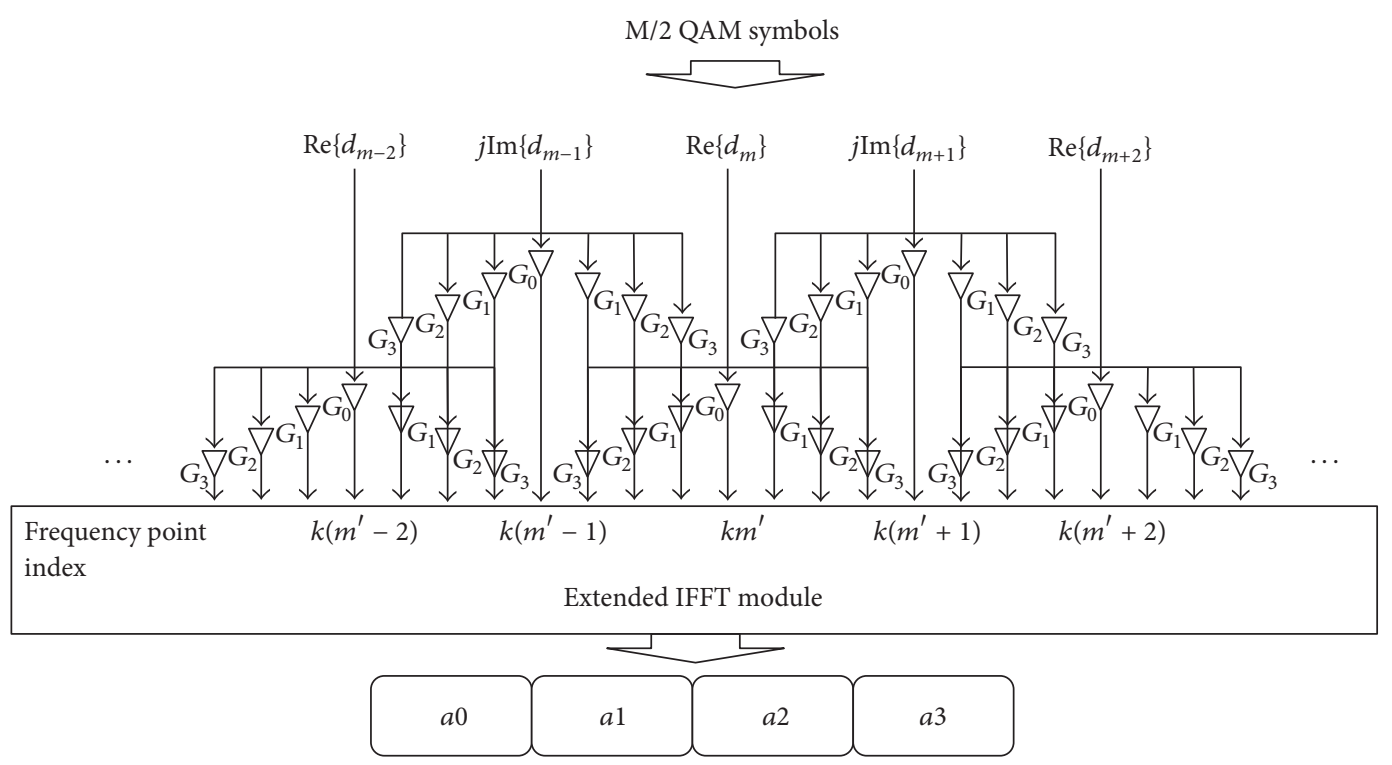

$M$ samples

FIgURE 2: Synthesis filter bank generation in FS-FBMC system $(K=4)$ [5].

prototype filter, the data can be reconstructed after removing the imaginary part in (2).

This process can be implemented in the frequency domain at the analysis filter bank in FS-FBMC system. The KM samples are taken by using a deoverlapping scheme, with these samples entering into the extended FFT of size $\mathrm{KM}$ module, as in Figure 3 . Then, the $2 K-1$ frequency points are merged with weight, according to the frequency coefficients $G_{|k|}$ of the prototype filter. This operation is called "frequency despreading" [5]. The data can be reconstructed after frequency despreading as follows:

$$
d_{m, n}+I_{m, n}=\frac{1}{K} \sum_{k=-K+1}^{K-1} F_{K m^{\prime}+k, n} G_{|k|}
$$

where $m^{\prime}$ is the index of frequency point, $F_{K m^{\prime}+k, n}$ is the result of extended-FFT process at each frequency point in the $m$ th subchannel in the $n$th FBMC symbol. This process can be depicted as Figure 3.

\section{The Proposed Channel Estimation Scheme for FS-FBMC System}

In the presence of channel distortion, the received FBMC/ OQAM signal can be expressed as follows if the transmission channel can be considered as flat during each subchannel:

$$
r(t)=\sum_{m=0}^{M-1} \sum_{n=0}^{N-1} d_{m, n} H_{m, n} g_{m, n}(t)+n(t),
$$

where $H_{m, n}$ is the transmission channel state information (CSI) at $g_{m, n}(t)$ and $n(t)$ is the white Gaussian noise.
After matched filtering in the analysis filter bank, the result can be represented as follows [8]:

$$
\begin{aligned}
r_{m, n} & =H_{m, n}\left(d_{m, n}+j \sum_{p, q \neq m, n} d_{p, q}\langle g\rangle_{m, n}^{p, q}\right)+n_{m, n} \\
& =H_{m, n}\left(d_{m, n}+I_{m, n}\right)+n_{m, n} .
\end{aligned}
$$

If the scattered pilot subchannel scheme is used for CSI estimation, the estimated CSI at the pilot location subchannel $\left(m_{p}, n_{p}\right)$ can be represented as follows [8]:

$$
\widehat{H}_{m_{p}, n_{p}}=\frac{r_{m_{p}, n_{p}}}{d_{m_{p}, n_{p}}}=\frac{H_{m_{p}, n_{p}}\left(d_{m_{p}, n_{p}}+I_{m_{p}, n_{p}}\right)+n_{m_{p}, n_{p}}}{d_{m_{p}, n_{p}}} .
$$

If the intrinsic interference for pilot subchannel $I_{m_{p}, n_{p}}$ is removed by using the interference cancelling schemes like $[8,11]$ and many other approaches, $\widehat{H}_{m_{p}, n_{p}}$ can be accepted to valid CSI estimation from the pilot subchannel. This process is conventional CSI estimation scheme using pilot subchannel in FBMC/OQAM system.

From here, the channel estimation scheme for FS-FBMC system using this CSI is introduced. In FS-FBMC system, the transmitted data can be reconstructed after equalizing process in the frequency domain for each frequency point and frequency despreading process, as follows [5]:

$$
d_{m, n}+I_{m, n}=\frac{1}{K} \sum_{k=-K+1}^{K-1} \frac{F_{K m^{\prime}+k, n}}{H_{K m^{\prime}+k, n}} G_{|k|},
$$

where $H_{K m^{\prime}+k, n}$ is CSI at each frequency point in the $m$ th subchannel in the $n$th FBMC symbol. This process can be depicted as Figure 4. 


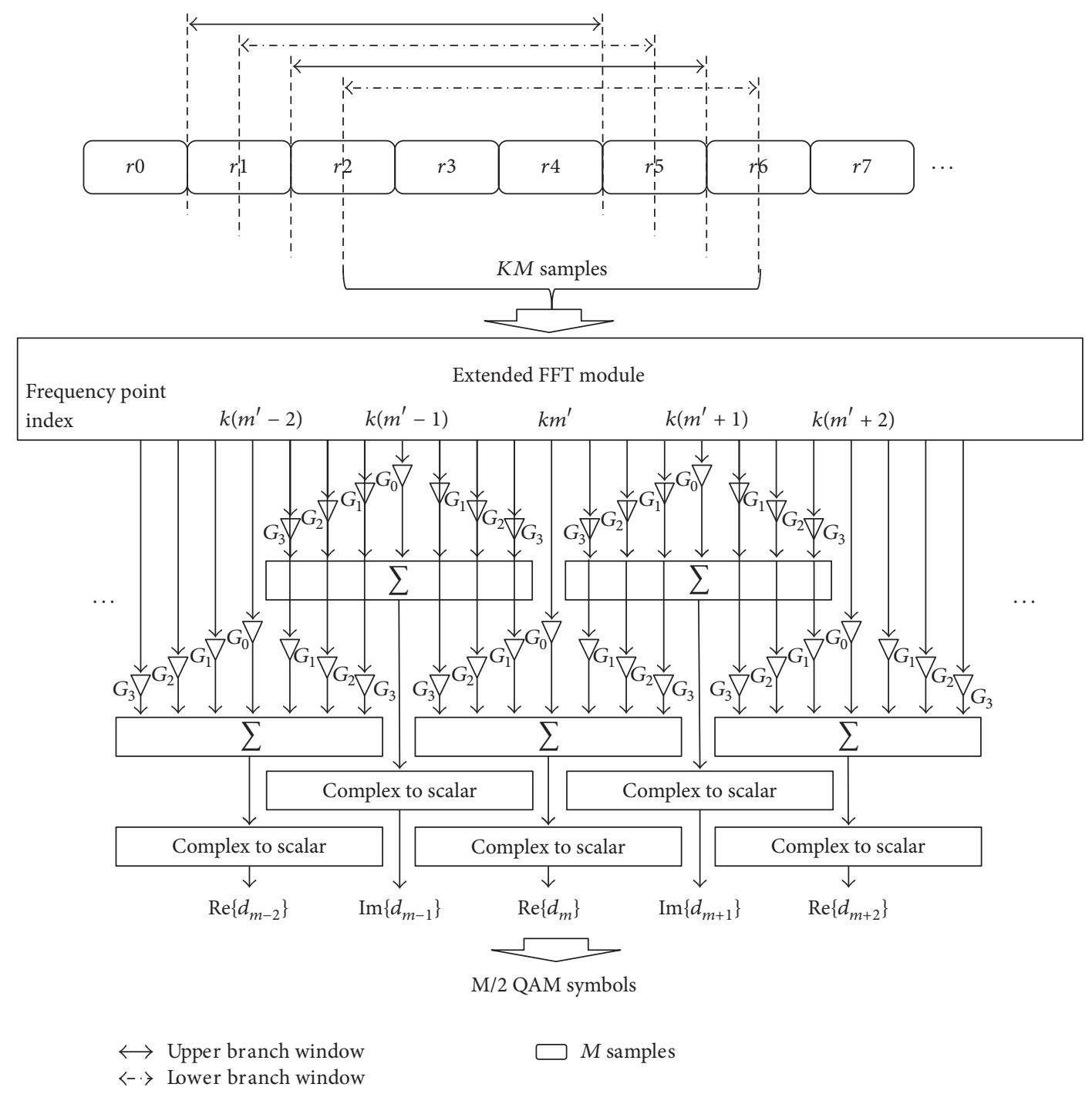

FIGURE 3: Deoverlapping and analysis filter bank generation in FS-FBMC system [5].

Therefore, the CSI estimation task for each frequency point is required for FS-FBMC system. First, the CSI estimation for each subchannel $\left(\ldots, \widehat{H}_{m-1, n}, \widehat{H}_{m, n}, \widehat{H}_{m+1, n}, \ldots\right)$ can be obtained after interpolation processes which have been proposed for channel estimation in OFDM/QAM systems [12-14]. After then, the CSI for each frequency point $\left(\ldots, \widehat{H}_{K\left(m^{\prime}-1\right)+k, n}, \widehat{H}_{K m^{\prime}+k, n}, \widehat{H}_{K\left(m^{\prime}+1\right)+k, n}, \ldots\right)$ for $(-K+1 \leq$ $k \leq K-1)$ can be obtained by interpolating again between the CSI of neighboring subchannels like Figure 5.

In this case, the CSI at the center frequency point for each subchannel $\left(\ldots, \widehat{H}_{K\left(m^{\prime}-1\right), n}, \widehat{H}_{K m^{\prime}, n}, \widehat{H}_{K\left(m^{\prime}+1\right), n}, \ldots\right)$ has to be obtained to interpolate other frequency points.

If the transmission channel is flat during subchannel, the CSI estimation using (6) is equivalent with the CSI at the center frequency point. Therefore, the CSI for each subchannel $\left(\ldots, \widehat{H}_{m-1, n}, \widehat{H}_{m, n}, \widehat{H}_{m+1, n}, \ldots\right)$ is substituted directly for center frequency point $\left(\ldots, \widehat{H}_{K\left(m^{\prime}-1\right), n}, \widehat{H}_{K m^{\prime}, n}, \widehat{H}_{K\left(m^{\prime}+1\right), n}, \ldots\right)$ at first CSI interpolation.
However, if the transmission CSI cannot be considered as flat during each subchannel, this CSI for each subchannel cannot be ensured to be equivalent with the center frequency point. In this case, this estimated CSI has to be modified to improve the performance of channel equalization task.

One way to check the reliability of estimated CSI is to compare the reproduced pilot at the receiver with the transmitted pilot signal. If the CSI is estimated perfectly for every frequency point in a pilot subchannel, the result of (8) for a pilot subchannel is equivalent to the amplitude of the pilot symbol.

$$
\widehat{d}_{m_{p}, n_{p}}=\frac{1}{K} \sum_{k=-K+1}^{K-1} \frac{F_{K m_{p}^{\prime}+k, n_{p}}}{\widehat{H}_{K m_{p}^{\prime}+k, n_{p}}} G_{|k|} .
$$

In order to make the CSI modification process as straightforward calculation, the following supposition is employed 


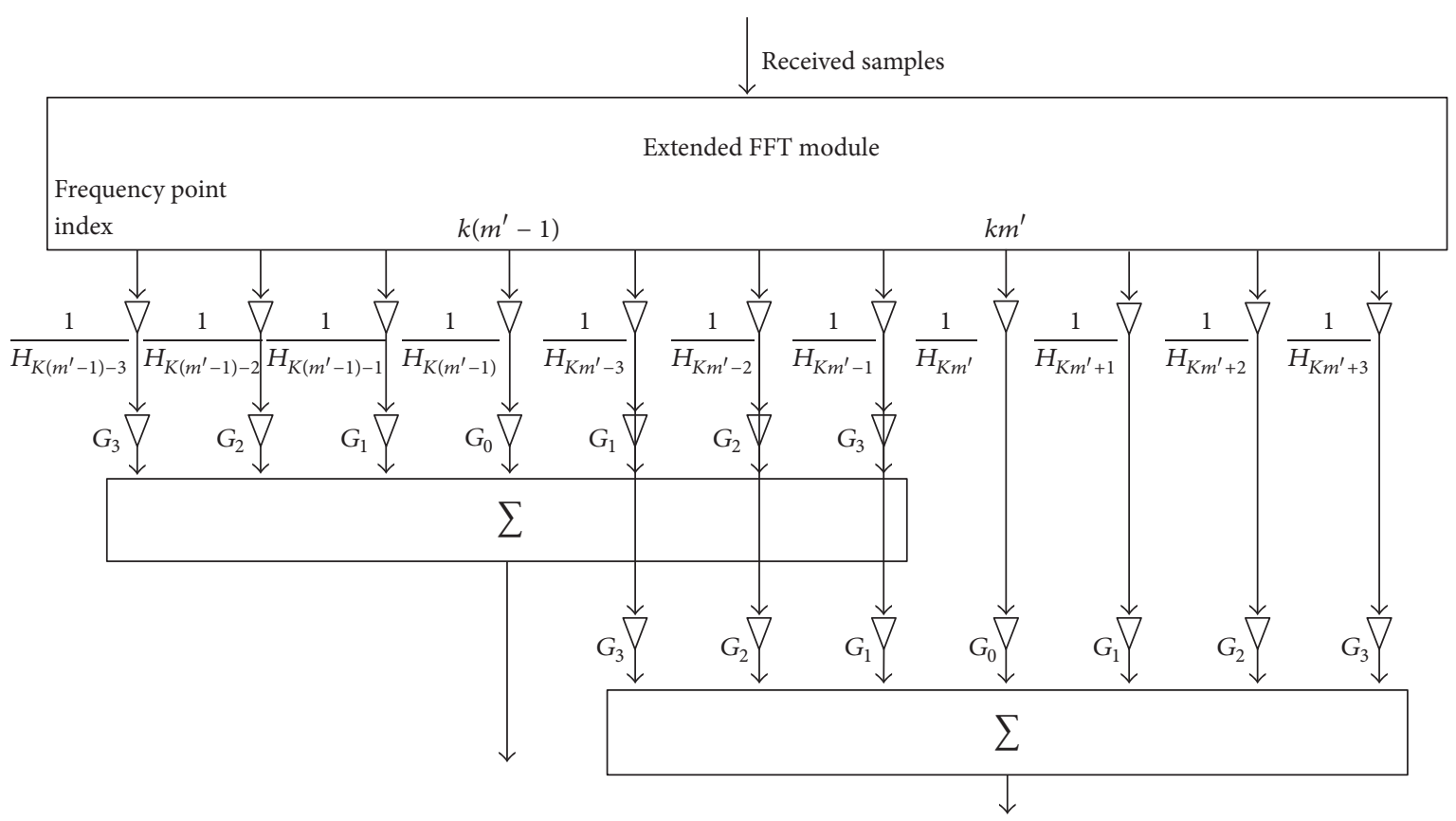

FIGURE 4: Channel equalizer for FS-FBMC system.

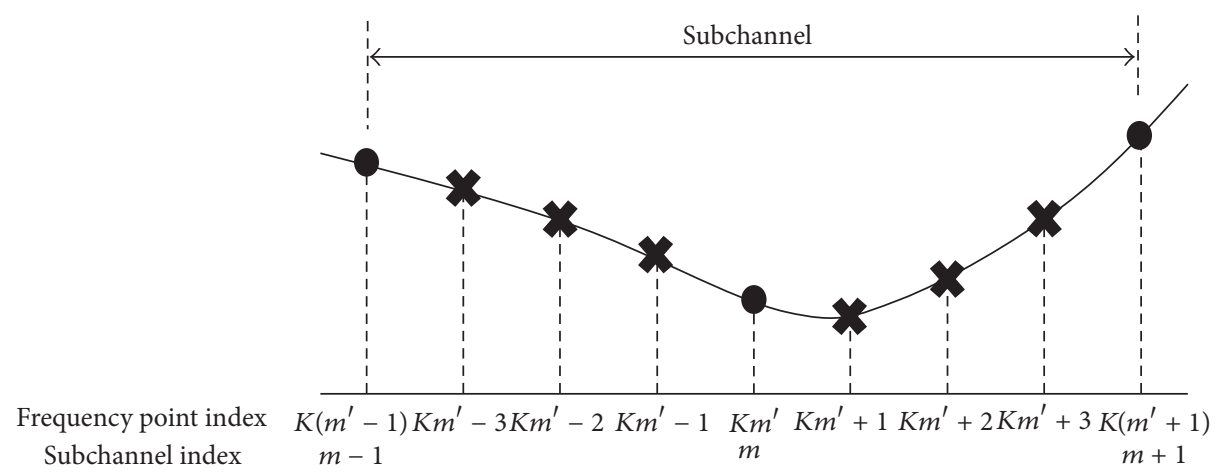

FIGURE 5: Channel state information estimation for frequency points in a subchannel.

for further process. Supposing that the ratio between estimated CSI $\widehat{H}_{K m_{p}+k, n_{p}}$ and practical CSI $H_{K m_{p}+k, n_{p}}$ for $(-K+$ $1 \leq k \leq K-1)$ is consistent, the CSI for each frequency point in the pilot subchannel can be obtained as follows by combining (7) and (8):

$$
\begin{aligned}
H_{K m_{p}^{\prime}+k, n_{p}}=\widehat{H}_{K m_{p}^{\prime}+k, n_{p}} \frac{\widehat{d}_{m_{p}, n_{p}}}{d_{m_{p}, n_{p}}}, & \\
& (k=-K+1, \ldots, K-1) .
\end{aligned}
$$

In fact, the ratio between $\widehat{H}_{K m_{p}^{\prime}+k, n_{p}}$ and CSI $H_{K m_{p}^{\prime}+k, n_{p}}$ for $(-K+1 \leq k \leq K-1)$ cannot be consistent perfectly for every frequency point during the subchannel. However, if the CSI interpolation scheme can reflect the variation of transmission channel during each subchannel in some degree, this process can reduce the estimation error between $\widehat{H}_{K m_{p}^{\prime}+k, n_{p}}$ and $H_{K m_{p}^{\prime}+k, n_{p}}$. Therefore, the estimated CSI can be modified iteratively as follows:

$$
\widehat{H}_{m_{p}, n_{p}}^{i+1}=\widehat{H}_{m_{p}, n_{p}}^{i} \frac{\widehat{d}_{m_{p}, n_{p}}^{i}}{d_{m_{p}, n_{p}}},
$$

where $i$ is the iteration stage of (10); $\widehat{H}_{m_{p}, n_{p}}^{i}$ is the $i$ th CSI at the pilot subchannel; and $\widehat{d}^{i}$ is the $i$ th estimated value of each pilot subchannel location using (7).

A flow chart of proposed channel estimation algorithm is depicted in Figure 6. The channel estimation scheme is performed by the following process. At first, the estimated CSI at the pilot subchannel location can be extracted after the frequency despreading and pilot extraction process. And this CSI is interpolated for each frequency point. Then, this CSI for each frequency point is used for equalization of received samples, as in Figure 4. Finally, the CSI for pilot subchannel 


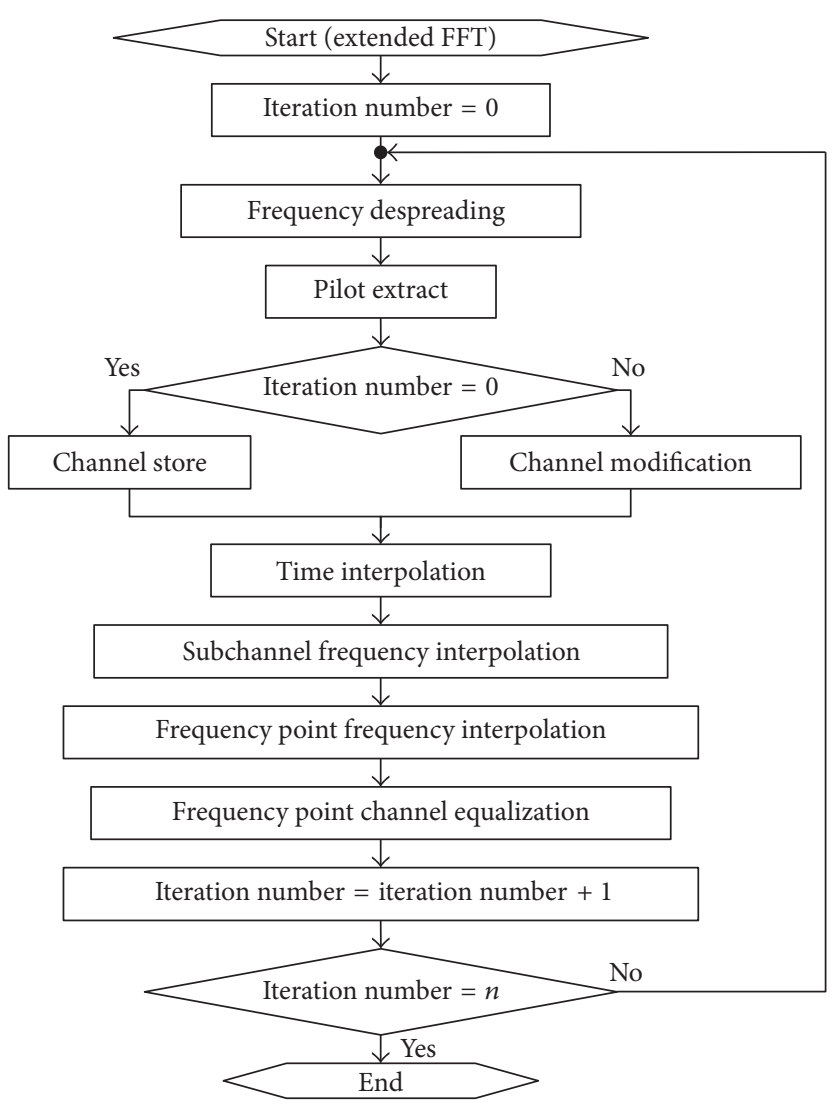

FIgURE 6: Flow chart of proposed channel estimation and equalization algorithm.

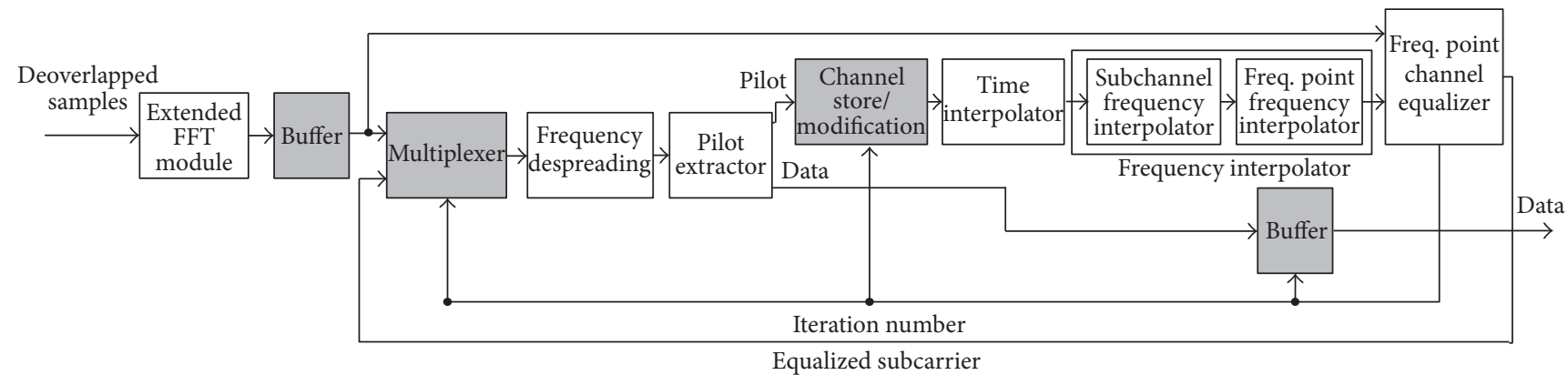

FIGURE 7: Block diagram of proposed channel estimator and equalizer of FS-FBMC system.

is modified by (10). These processes are performed iteratively for predefined iteration number.

A block diagram of the proposed channel estimator is depicted in Figure 7. The CSI for every stage is stored in the channel store/correction block, interpolated in the time and frequency interpolator to obtain the CSI about each frequency point. Then, the frequency equalization for each frequency point is performed in frequency point channel equalizer, as in (7). After then, the CSI modification is performed at the channel store/modification block, with the pilot estimation value as in (10).
If we classify the components in Figure 7 into two sections to discuss the implementation for the proposed channel estimator, the white boxes are common blocks necessary for the implementation of FS-FBMC system while the shaded boxes are only the necessary block for proposed scheme. In the case of common blocks necessary for FS-FBMC system, the analyses of implementation issue are discussed in [15, 16]. Thus in the complexity sense, the increased hardware includes memory blocks for buffering, multiplexer for input data for channel store/modification block, and multiplier for CSI estimation modifying process for each subchannel. The 


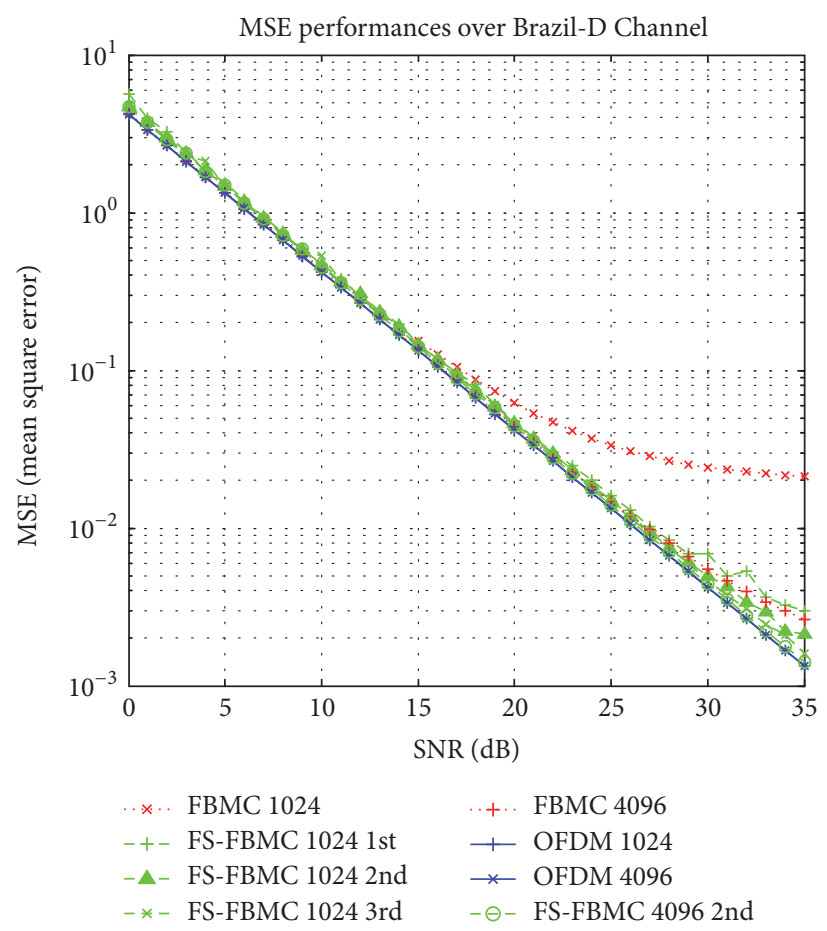

FIGURE 8: MSE comparison between the estimated CSI and transmission CSI at pilot subchannel.

TABLE 1: Simulation parameters.

\begin{tabular}{ll}
\hline Parameter & Value \\
\hline The number of subchannels & 256 or 1,024 or 4,096 \\
Constellation mapping & 256 QAM \\
Sampling period $[\mu \mathrm{s}]$ & $0.1458(=7 / 48)$ \\
Pilot spacing in frequency domain (subchannels) & 2 for 256 subchannels \\
Pilot spacing in time domain (subchannels) & 4 for $1,024 / 4,096$ subchannels \\
CSI interpolation method in frequency domain & 8 (for 256/1,024/4,096 subchannels) \\
CSI interpolation method in time domain & Cubic spline interpolation method \\
Equalization method & Linear interpolation method \\
Brazil-D Channel path delay $[\mu \mathrm{s}]$ & Zero forcing method \\
Brazil-D Channel path power $[\mathrm{dB}]$ & {$[0.15,0.63,2.22,3.05,5.86,5.93]$} \\
Brazil-D fading model & {$[-0.1,-3.8,-2.6,-1.3,0.0,-2.8]$} \\
\hline
\end{tabular}

required memory block and multiplier can be reduced at the expense of increased operation frequency of channel estimator by using resource sharing.

\section{Simulation Results}

We have simulated the CSI estimation performance and symbol error rate (SER) with the parameters listed in Table 1.

First, the mean square error (MSE) between estimated CSI and original channel CSI is computed at all pilot subchannels. Figure 8 shows the MSE values for various SNR situation. It is easily known that the CSI estimation performance using (6) is severely affected by the frequency selectivity (related to the number of subchannels). On the other hand, the proposed channel estimation operating in the frequencyspreading domain is insensitive to the frequency selectivity. Furthermore, the estimation performance is improved as the number of iteration increases.

Figures 9(a)-9(c) show the comparison of channel compensated SER performances for Brazil-D Channel between FS-FBMC with proposed iterative channel compensation scheme and conventional OFDM/QAM system. Solid red indicates the SER for the subcarrier channel compensation performance using (6); dotted green is the SER for the proposed FS-FBMC channel compensation with increased iteration. This simulation shows that SER performances can be improved by equalizing every frequency point, respectively, after obtaining the CSI using interpolation method. 


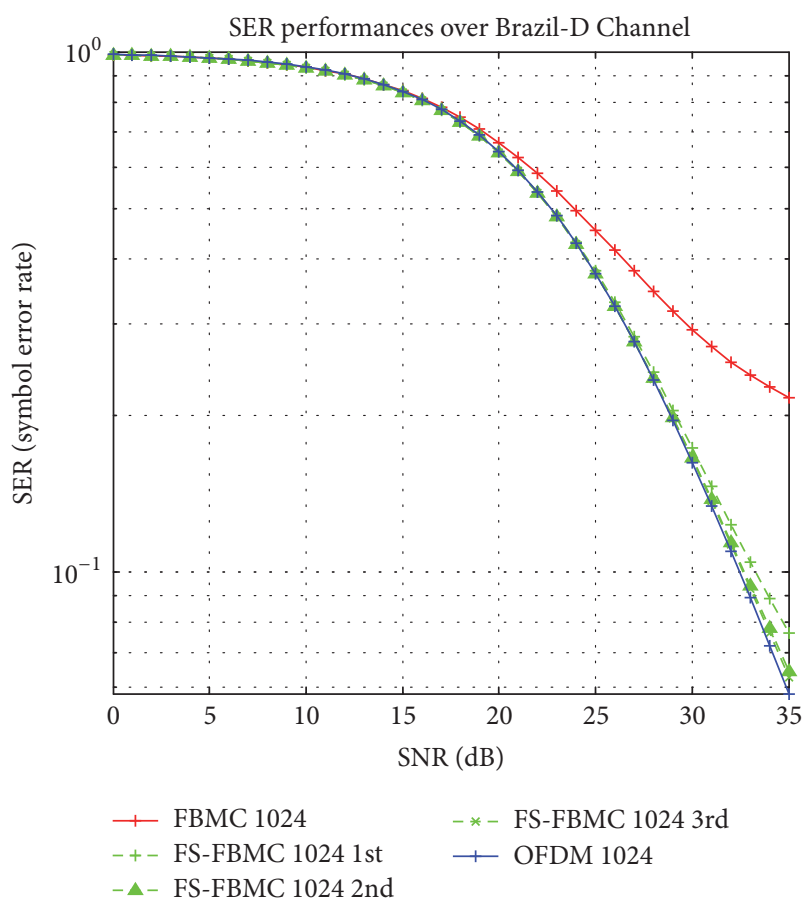

(a)

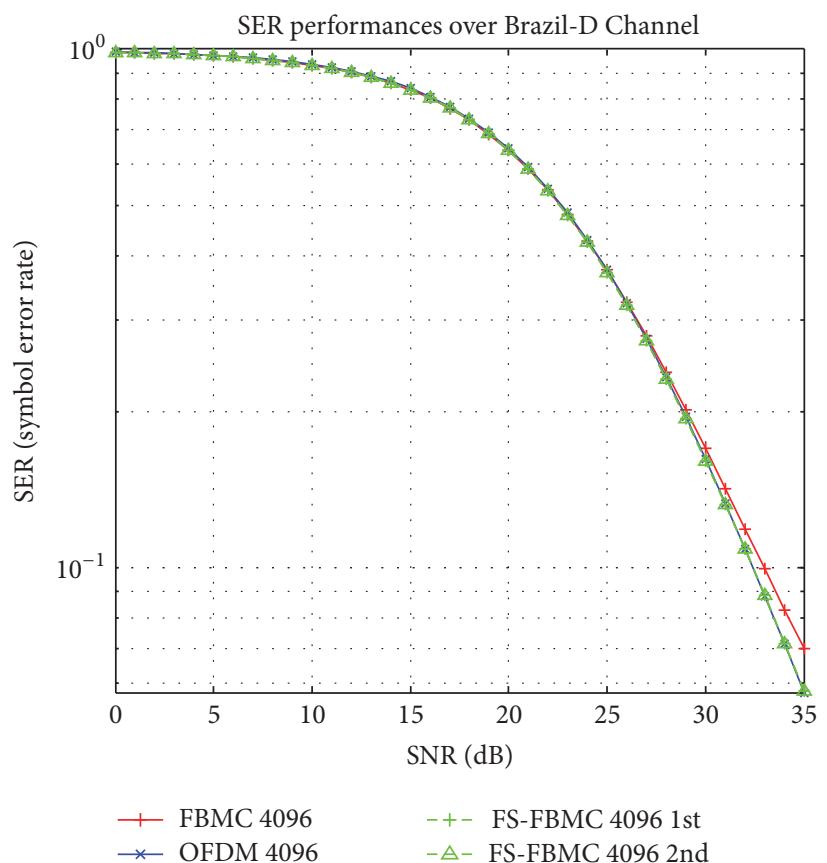

(b)

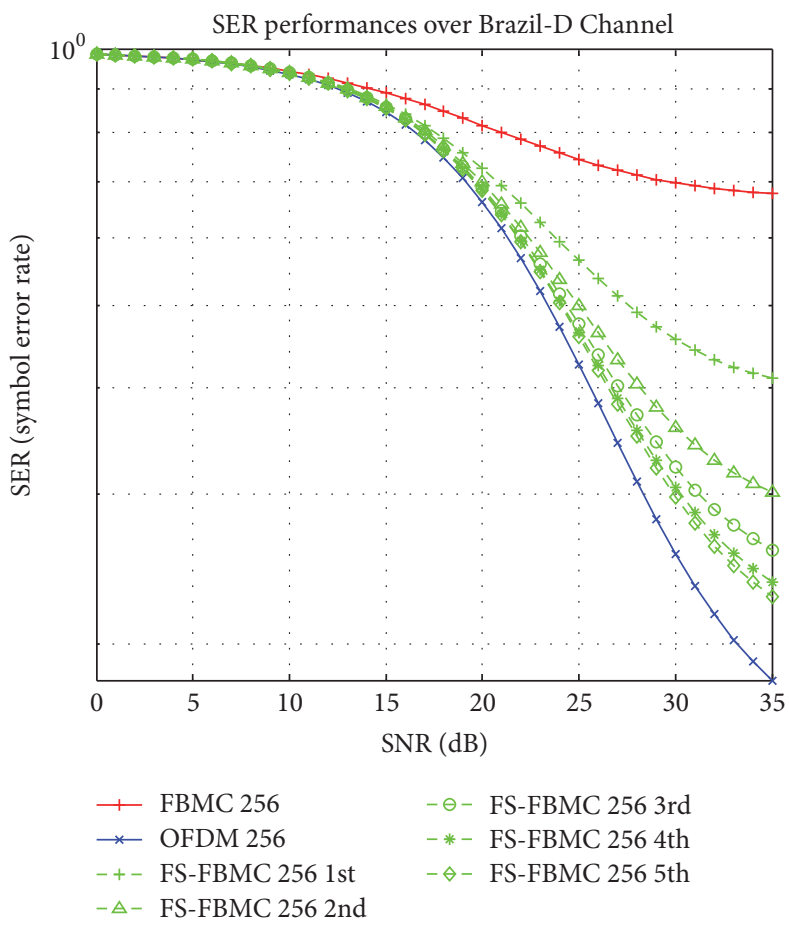

(c)

FIgURE 9: (a) SER performances over Brazil-D Channel profile (1,024 subchannels). (b) SER performances over Brazil-D Channel profile (4,096 subchannels). (c) SER performances over Brazil-D Channel profile (256 subchannels).

Moreover, this SER can be further improved as the CSI is modified iteratively by using the proposed estimation scheme.

Additionally, we have simulated 256 subchannels in Brazil-D Channel profile. In this case, the maximum delay of the simulation channel is about $5.9 \mu \mathrm{sec}$ and one OQAM time offset for 256 subchannels is $18.7 \mu$ sec showing a little poor performance when directly compared to the large subchannel environment such as 4,096 subchannels. However, the proposed channel estimation scheme can improve the SER performance as the number of iterations increases. 


\section{Competing Interests}

The authors declare that there is no conflict of interests regarding the publication of this paper.

\section{Acknowledgments}

This work was supported by ICT R\&D program of MSIP/IITP [R0101-16-0189, development of the next generation convergence broadcasting and monitoring systems combined with the networks].

\section{References}

[1] W. Y. Zou and Y. Wu, "COFDM: an overview," IEEE Transactions on Broadcasting, vol. 41, no. 1, pp. 1-8, 1995.

[2] F. Khan, LTE for 4G Mobile Broadband: Air Interface Technologies and Performance, Cambridge University Press, 2009.

[3] Digital Video Broadcasting (DVB), "Frame structure channel coding and modulation systems for a second generation digital terrestrial television broadcasting system(DVB-T2)," ETSI EN 302755 v1.3.1 2011-11, 2011.

[4] H. Bölcskei, P. Duhamel, and R. Hleiss, "Design of pulse shaping OFDM/OQAM systems for high data-rate transmission over wireless channels," in Proceedings of the IEEE International Conference on Communications (ICC '99), vol. 1, pp. 559-564, Vancouver, Canada, June 1999.

[5] M. G. Bellanger, FBMC Physical Layer: A Primer, PHYDYAS, 2010.

[6] J. Du and S. Signell, "Classic OFDM systems and pulse-shaping OFDM/OQAM systems," Tech. Rep., KTH-Royal Institute of Technology, 2007.

[7] P. Siohan, C. Siclet, and N. Lacaille, "Analysis and design of OFDM/OQAM systems based on filterbank theory," IEEE Transactions on Signal Processing, vol. 50, no. 5, pp. 1170-1183, 2002.

[8] J.-P. Javaudin, D. Lacroix, and A. Rouxel, "Pilot-aided channel estimation for OFDM/OQAM," in Proceedings of the 57th IEEE Semiannual Vehicular Technology Conference (VTC '03), vol. 3, pp. 1581-1585, April 2003.

[9] M. G. Bellanger and J. L. Daguet, "TDM-FDM transmultiplexer: digital polyphase and FFT," IEEE Transactions on Communications, vol. 22, no. 9, pp. 1199-1205, 1974.

[10] M. Bellanger, "FS-FBMC: a flexible robust scheme for efficient multicarrier broadband wireless access," in Proceedings of the 2012 IEEE Globecom Workshops (GC Wkshps '12), pp. 192-196, Anaheim, Calif, USA, December 2012.

[11] C. Lélé, R. Legouable, and P. Siohan, "Channel estimation with scattered pilots in OFDM/OQAM," in Proceedings of the IEEE 9th Workshop on Signal Processing Advances in Wireless Communications (SPAWC'08), pp. 286-290, Recife, Brazil, July 2008.

[12] S. Coleri, M. Ergen, A. Puri, and A. Bahai, "Channel estimation techniques based on pilot arrangement in OFDM systems," IEEE Transactions on Broadcasting, vol. 48, no. 3, pp. 223-229, 2002.

[13] I. Gutiérrez, J. L. Pijoan, F. Bader, and R. Aquilué, "New channel interpolation method for OFDM systems by nearest pilot padding," in Proceedings of the 12th European Wireless Conference on Enabling Technologies for Wireless Multimedia Communications, European Wireless, Athens, Greece, April 2006.

[14] P.-Y. Tsai and T.-D. Chiueh, "Frequency-domain interpolationbased channel estimation in pilot-aided OFDM systems," in
Proceedings of the IEEE 59th Vehicular Technology Conference (VTC-Spring '04), vol. 1, pp. 420-424, Milan, Italy, May 2004.

[15] V. Berg, J.-B. Dore, and D. Noguet, "A flexible FS-FBMC receiver for dynamic access in the TVWS," in Proceedings of the 9th International Conference on Cognitive Radio Oriented Wireless Networks (CROWNCOM '14), pp. 285-290, Oulu, Finland, June 2014.

[16] D. Mattera, M. Tanda, and M. Bellanger, "Frequency-spreading implementation of OFDM/OQAM systems," in Proceedings of the 9th International Symposium on Wireless Communication Systems (ISWCS '12), pp. 176-180, August 2012. 


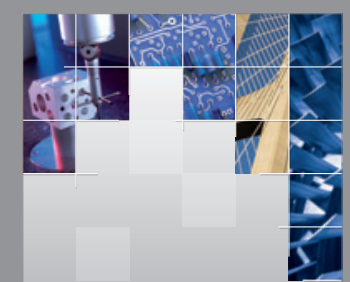

\section{Enfincering}
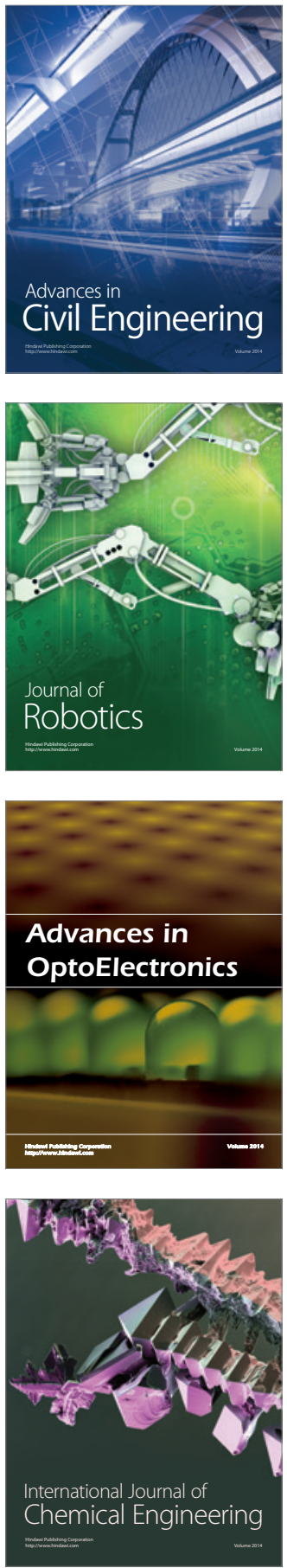

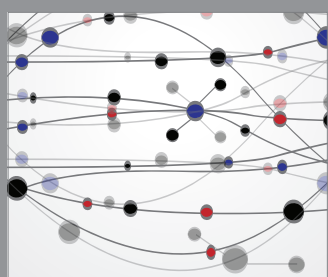

The Scientific World Journal

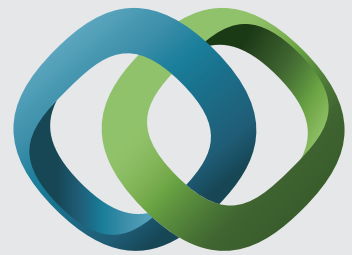

\section{Hindawi}

Submit your manuscripts at

https://www.hindawi.com
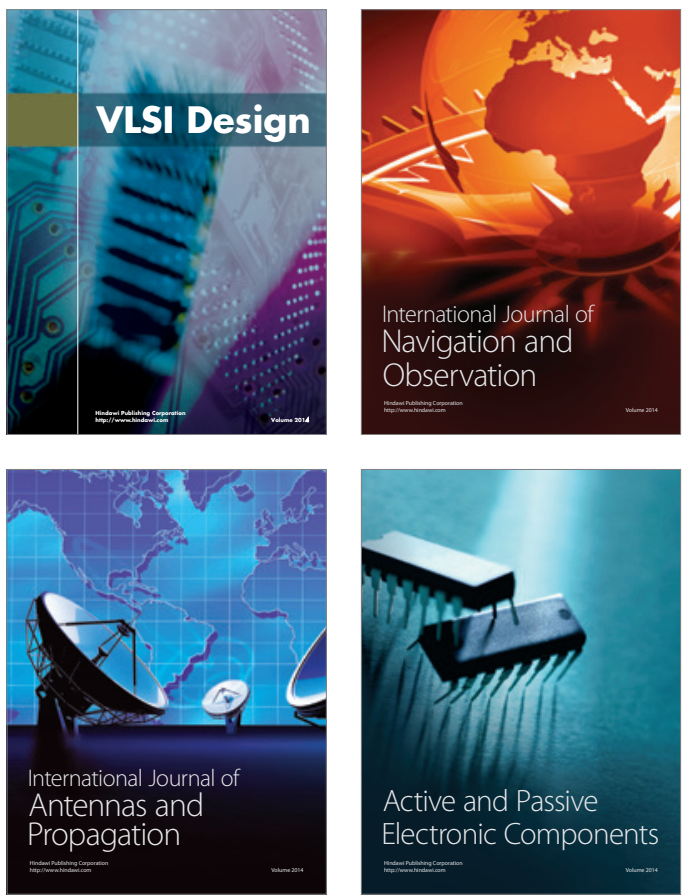
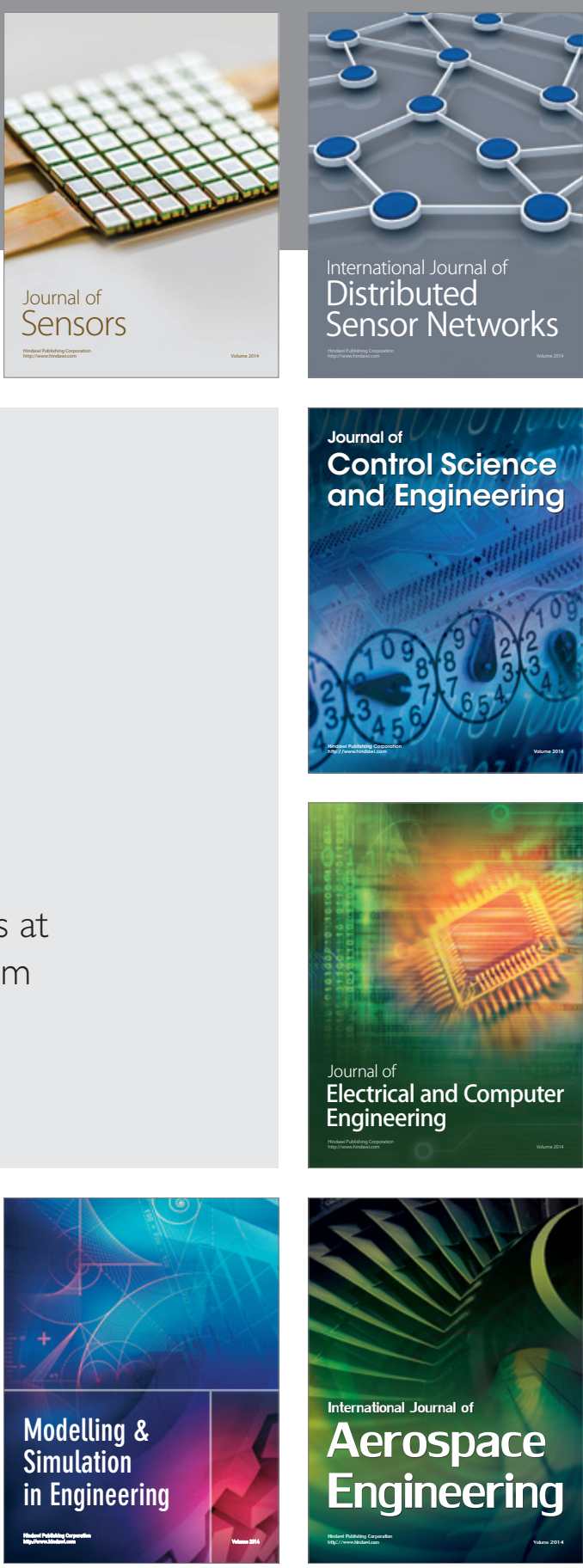

International Journal of

Distributed

Sensor Networks

$-$

Joumal of

Control Science

and Engineering
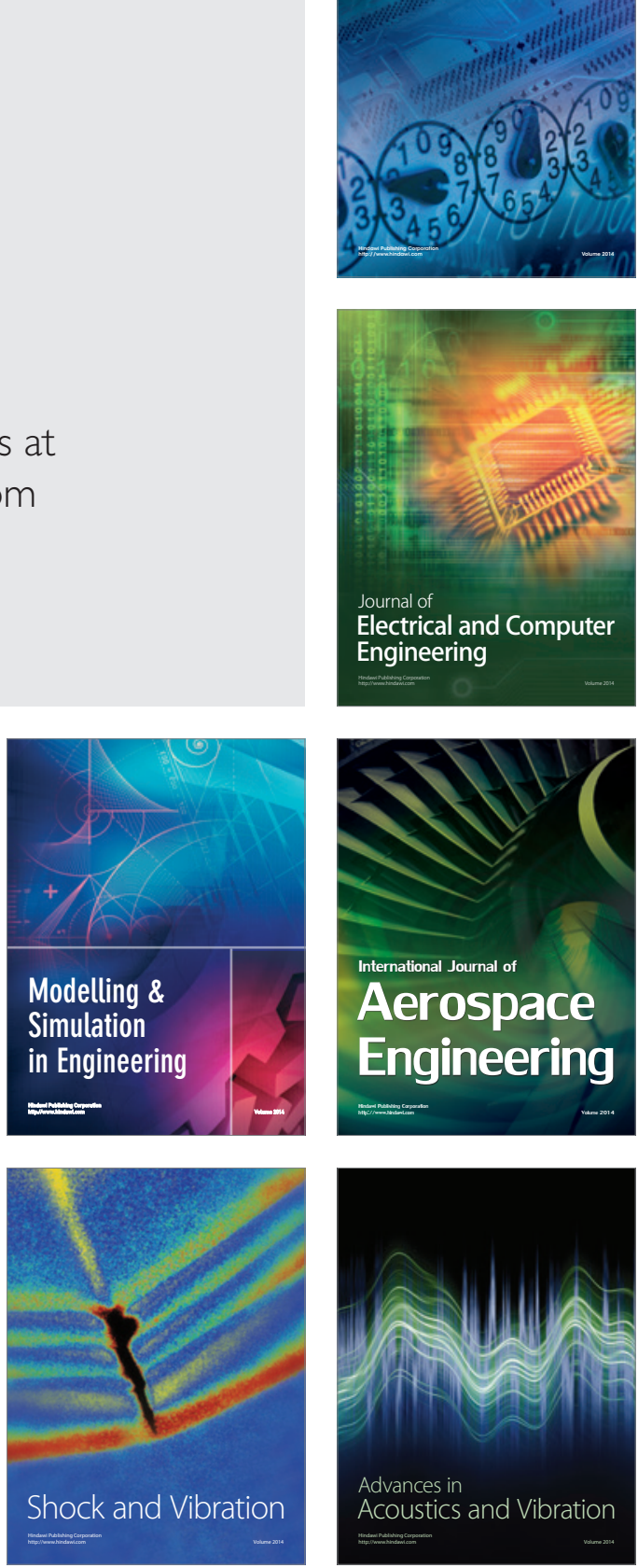